\title{
Oral budesonide is as effective as oral prednisolone in active Crohn's disease
}

\author{
M Campieri, A Ferguson, W Doe, T Persson, L-G Nilsson, and the Global Budesonide \\ Study Group
}

\begin{abstract}
Background-The use of corticosteroids in active Crohn's disease often becomes limited by side effects. Budesonide is a potent corticosteroid with low systemic bioavailability due to an extensive first pass liver metabolism.

Aims-To compare the efficacy and safety of two dosage regimens of budesonide and prednisolone in patients with active Crohn's disease affecting the ileum and/or the ascending colon.

Patients and methods-One hundred and seventy eight patients were randomised to receive budesonide controlled ileal release (CIR) capsules $9 \mathrm{mg}$ once daily or $4.5 \mathrm{mg}$ twice daily, or prednisolone tablets $40 \mathrm{mg}$ once daily. The treatment period was 12 weeks. The primary efficacy variable was clinical remission, defined as a Crohn's Disease Activity Index (CDAI) of 150 or less.

Results-After eight weeks of treatment, remission occurred in $60 \%$ of patients receiving budesonide once daily or prednisolone and in $42 \%$ of those receiving budesonide twice daily $(p=0.062)$. The presence of glucocorticoid associated side effects was similar in all groups; however, moon face was more common in the prednisolone group $(p=0.0005)$. The highest frequency of impaired adrenal function, as measured by a short ACTH test, was found in the prednisolone group $(\mathrm{p}=0.0023)$. Conclusions-Budesonide CIR, administered at $9 \mathrm{mg}$ once daily or $4.5 \mathrm{mg}$ twice daily, is comparable to prednisolone in inducing remission in active Crohn's disease. The single dose administration is as promptly effective as prednisolone and represents a simpler and safer therapeutic approach, with a considerable reduction in side effects.

(Gut 1997; 41: 209-214)
\end{abstract}

Keywords: adrenal function; CDAI; glucocorticoid; glucocorticoid associated side effects

Astra Draco AB, Lund, Sweden

T Persson

L-G Nilsson

Correspondence to: Professor M Campieri, Medical and

Gastroenterological Clinic,

University of Bologna,

Policlinico S Orsola, Via

Massarenti, 9, I-40138

Bologna, Italy.

Accepted for publication 23 January 1997

Crohn's disease is a chronic inflammatory disorder of unknown aetiology. Although any portion of the digestive tract from mouth to anus may be involved, the most commonly affected parts are the distal ileum and the ascending colon. ${ }^{1}$ To date, glucocorticoids (GCS)prednisone or prednisolone-have been the most effective drugs in inducing clinical remission in these patients with Crohn's disease ${ }^{2}$; unfortunately their therapeutic efficacy is counterbalanced by unwanted side effects attributable to their absorption and pharmacological (systemic) action or to their suppression of endogenous adrenal function. ${ }^{3}$ Moreover, in clinical practice it has often been difficult to wean patients off systemically active GCS without triggering a relapse of the disease. New GCS have been developed which possess potent topical anti-inflammatory activity and with a systemic activity less than conventional GCS. ${ }^{4}$ The unique therapeutic ratio of the new analogues is due to a high potency combined with their extensive and rapid first pass liver metabolism, where the metabolites have minimal or no GCS activity.

Budesonide is the most extensively studied compound of this new group of GCS. When administered by inhalation, budesonide has been found to be effective and safe in the treatment of both asthma and rhinitis. ${ }^{5}$ Given as an enema, it has also been found to be as effective as conventional GCS enemas in the treatment of distal ulcerative colitis but has the clear advantage of producing significantly less adrenal suppression than conventional GCS. ${ }^{6-9}$

Budesonide has also been developed in a gastric resistant formulation (Entocort ${ }^{\mathbb{Q}}$ capsules, Astra Draco, Lund, Sweden) containing pellets with slow release properties; this preparation allows the drug to be delivered mainly to the ileum and ascending colon. ${ }^{10}$ The properties of this formulation, together with the high GCS potency and extensive first pass liver metabolism of budesonide, offer improved therapy for Crohn's disease by reducing the risk of steroid associated side effects. In previous studies, ${ }^{11-13}$ budesonide controlled intestinal release (CIR) capsules $9 \mathrm{mg}$ daily were effective in inducing remission in patients with active Crohn's disease affecting the ileum and the ascending colon. In a placebo controlled dose finding study, ${ }^{12}$ budesonide CIR $4.5 \mathrm{mg}$ twice daily was found to be the lowest effective dose, while in a study designed to compare budesonide $9 \mathrm{mg}$ once daily and prednisolone $40 \mathrm{mg},{ }^{13}$ both agents were equally effective in inducing remission.

However, prednisolone reduced the mean
Crohn's Disease Activity Index (CDAI) scores significantly more, whereas budesonide $9 \mathrm{mg}$ once daily gave rise to significantly fewer glucocorticoid associated side effects and less suppression of endogenous cortisol production. It was felt important to study further the clinical efficacy of budesonide and the impact on the adrenal glands in comparison with 
prednisolone, and whether there were any differences if budesonide was given once or twice daily.

\section{Methods}

SELECTION OF PATIENTS

Twenty six investigational centres in the United Kingdom, Ireland, Italy, Australia, New Zealand, Germany, Sweden, Belgium, and The Netherlands participated in the study.

Eligible patients were older than 18 years of age, with a confirmed diagnosis of active Crohn's disease, as defined by a score of 200 or higher on the CDAI. ${ }^{14}$ The extent of disease had to be defined within 24 months before randomisation; entry was restricted to patients with disease involving the ileum and/or the ascending colon but not extending beyond the hepatic flexure. Patients who had undergone ileostomy or more extensive resection of the ileum $(>100 \mathrm{~cm})$, and those with severe disease requiring imminent surgery, were not enrolled in the study. They were not eligible if they had complications including abscesses, perforations, or active fistulas. Patients with concomitant active peptic ulcer or clinically important hepatic, renal, cardiovascular, or psychiatric conditions were also excluded. Immunosuppressive drugs were allowed until three months before the study, 5-aminosalicylates and metronidazole until the day before the study, and corticosteroids allowed until one week before the study. The trial was performed in accordance with the Declaration of Helsinki and was approved by the Ethics Committees at all centres; all patients gave written or oral informed consent.

\section{STUDY DESIGN}

The trial was a randomised double blind, double dummy study. A baseline CDAI was obtained during a run-in period of three to seven days. The patients were subsequently randomised to treatment with either budesonide CIR capsules $9 \mathrm{mg}$ once daily or $4.5 \mathrm{mg}$ twice daily or prednisolone $40 \mathrm{mg}$ once daily. Budesonide CIR was tapered to $6 \mathrm{mg}$ after eight weeks and to $3 \mathrm{mg}$ after a further two weeks. Prednisolone was tapered to $30 \mathrm{mg}$ after two weeks and then continuously throughout the study, reaching $5 \mathrm{mg}$ after nine weeks. The $5 \mathrm{mg}$ dose was then continued for three weeks so that the total treatment period was 12 weeks. Follow up visits were carried out after two, four, eight, and 12 weeks of treatment.

STUDY DRUGS

The controlled ileal release gelatine capsules containing 3 or $1.5 \mathrm{mg}$ budesonide used in the study (Entocort ${ }^{\mathbb{E}}$ capsules) and placebo capsules were manufactured by Astra Draco AB (Lund, Sweden). The prednisolone tablets, 5 and $10 \mathrm{mg}$, and placebo tablets were obtained from As Hydro Pharma (Elverum, Norway). The drugs were provided in identical blister packages. Compliance was checked by the study personnel by counting unopened blisters. Patients were considered non-compliant if they consumed less then $75 \%$ of the study drugs during their actual treatment period or if they interrupted the study drugs for more than five consecutive days.

CLINICAL ASSESSMENT

At entry, patients' demographic characteristics, relevant current and past diagnoses, current medication, and history of previous bowel surgery were recorded. The distal part of the colon was assessed by sigmoidoscopy to exclude inflammation in the rectum. Disease extent was confirmed by endoscopy or radiology assessment if not done within the 24 months prior to the first visit.

CDAI was the main clinical assessment for determination of drug efficacy and it was calculated at the randomisation visit and at all subsequent visits. Remission was defined as a CDAI of 150 or less. The patients were provided with diary cards for all weeks of the study. On these, they recorded (each evening) the number of stools, general well being, abdominal pain, and intake of study medication. Adverse events were also recorded at each visit, as responses to a standard question ("Have you had any health problems or symptoms not usually associated with your bowel disorder since the last visit?"). Scores from the seven days preceding the clinic visit were used for the CDAI calculation.

The following analyses were done at each visit and used as measures of inflammation: erythrocyte sedimentation rate (ESR), platelet particle concentration, serum C-reactive protein (CRP) (before treatment and after four and 12 weeks), and serum orosomucoid.

Safety assessments consisted of the recording of any symptoms, clinical and haematological measurements, and an examination by the investigator for corticosteroid associated side effects. Blood samples for plasma cortisol analysis were drawn between 7.30 and $9.30 \mathrm{am}$, always at the same time on each occasion.

SHORT ACTH TEST

The responses to the short ACTH test (Synacthen $^{\circledR}$, Ciba-Geigy), at randomisation and after eight weeks of treatment, were analysed with regard to plasma cortisol concentrations before and 30 minutes after the ACTH injection; the magnitude of the increase was determined. Plasma cortisol concentration was analysed both at the centre and at Astra Draco AB. The analyses carried out at each centre were used only for safety purposes, whereas the results from analyses done at Astra Draco $A B$, using an HPLC method, ${ }^{15}$ are reported here. The adrenal function was considered normal if the 0 -minute plasma cortisol level was $\geqslant 150 \mathrm{nmol} / 1$ and either the plasma cortisol increase was $\geqslant 200 \mathrm{nmol} / 1$ or the 30-minute plasma cortisol concentration was $\geqslant 400 \mathrm{nmol} / 1$.

STATISTICAL ANALYSIS

From the National Cooperative Crohn's Disease Study (NCCDS) and other reports it was estimated that the remission rates after 10 weeks would reach $70 \%$ in the prednisolone 
group. ${ }^{14} 1617$ No or little difference in efficacy between the two budesonide regimens was assumed, while there might possibly be a difference between either of the budesonide regimens and prednisolone. The primary aim of this study was to assess the remission rates after two, eight, and 12 weeks of treatment. With 50 patients per group there was an $80 \%$ probability of detecting a significant difference if the budesonide remission rate was $40 \%$. A $95 \%$ confidence interval for the difference in remission rates between any two groups would have an uncertainty of $\pm 19 \%$. In order to compensate for non-evaluable patients, it was estimated that 180 randomised patients would be required. The analyses were based on data for all patients treated and the last available value after the baseline value. No correlations for multiple comparisons have been made.

\section{Results}

PATIENT ENROLMENT

A total of 178 patients were randomised and 177 were treated; 58 patients received budesonide $9 \mathrm{mg}$ once daily, 61 budesonide $4.5 \mathrm{mg}$ twice daily, and 58 received prednisolone. The demography and disease history for all patients treated, recruited at 26 centres, are presented in table 1 . The groups were well matched. Out of the 177 patients treated in the study, 36 prematurely discontinued their treatment.

The major reason (15\%) for treatment withdrawal was disease deterioration or no improvement (therapeutic failure). The frequencies of therapeutic failure observed were comparable in the three groups - that is, $16 \%$ in the budesonide once daily group, $16 \%$ in the budesonide twice daily group, and $12 \%$ in the prednisolone group. A $\chi^{2}$ test showed no significant differences between the treatment groups $(\mathrm{p}=0.78)$.

\section{CLINICAL EFFICACY}

Remission rates

Statistical evaluation of all patients treated showed that after two weeks of treatment the highest remission rate of $48 \%$ was observed in the budesonide once daily group, compared with $37 \%$ in the prednisolone group, and $27 \%$ in the budesonide twice daily group (fig 1). These differences in remission rates were not significant $(p=0.052)$. After eight weeks treatment, equal remission rates of $60 \%$ were found in the budesonide once daily and prednisolone groups, compared with $42 \%$ in the budesonide twice daily group (fig 1). The differences between the three groups were not statistically significant $(\mathrm{p}=0.062)$.

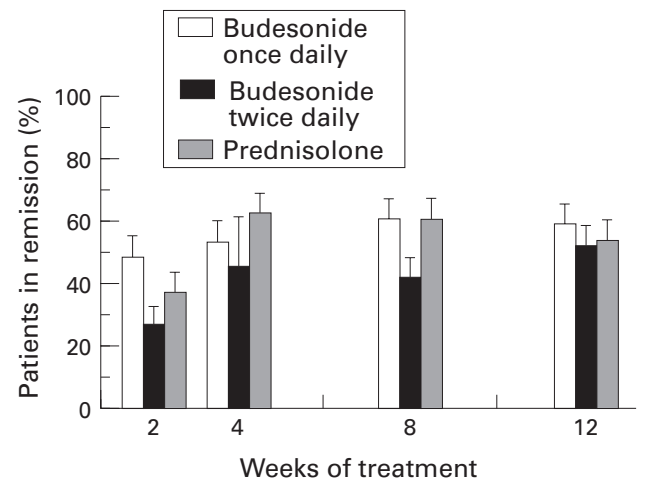

Figure 1: Mean (SE) proportion of patients in remission after two, four, eight, and 12 weeks of treatment with budesonide or prednisolone.

Analyses with respect to prognostic factors Analyses of remission rates by two-way analysis of variance were also performed with respect to the following prognostic factors:

- disease activity at inclusion (CDAI $\geqslant 300 /$ CDAI <300)

- previous bowel resection (yes/no)

- $\operatorname{sex}$

- previous steroid treatment during the past year (yes/no).

After eight weeks of treatment patients admitted to the study with a CDAI $<300$ showed an overall remission rate significantly higher than patients who entered with a CDAI >300. Of the patients admitted with a CDAI $<300$, remission was achieved in $31 / 44$ in the budesonide once daily group, in $21 / 40$ in the budesonide twice daily group, and in $22 / 44$ in the prednisolone group. In the group with a CDAI $\geqslant 300$, remission was achieved in $4 / 13$, $3 / 18$, and $7 / 13$ in the budesonide once daily, budesonide twice daily, and prednisolone groups, respectively. Disease activity was a prognostic factor which significantly $(p=0.0007)$ influenced the remission rates; however, the difference between treatments did not depend on the disease activity. Furthermore, the absolute decrease in mean CDAI was largest in the budesonide once daily group, irrespective of severity at entry.

There was a statistically significant interaction between treatment and the presence or absence of previous resection $(\mathrm{p}=0.030)$; although the remission rate was higher among non-resected patients in both the budesonide once daily group and the prednisolone group, the rate was higher among resected patients in the budesonide twice daily group. Remission rates for male or female patients, or for patients who had or did not have previous steroid treat-

TABLE 1 Demographic characteristics and disease history

\begin{tabular}{|c|c|c|c|c|c|c|}
\hline & \multicolumn{2}{|c|}{ Budesonide once daily $(n=58)$} & \multicolumn{2}{|c|}{ Budesonide twice daily $(n=61)$} & \multicolumn{2}{|c|}{ Prednisolone $(n=58)$} \\
\hline & Mean & Range & Mean & Range & Mean & Range \\
\hline Sex ratio $(M / F)$ & & $21 / 37$ & & $28 / 33$ & $23 / 35$ & \\
\hline Age (years) & 36 & $17-71$ & 38 & $20-71$ & 36 & $19-70$ \\
\hline Weight $(\mathrm{kg})$ & 63 & $41-118$ & 63 & $35-94$ & 61 & 39-93 \\
\hline CDAI & 277 & $121-476$ & 274 & $107-465$ & 279 & $202-458$ \\
\hline Disease duration (years) & 8.3 & $0-30$ & 7.9 & $0-37$ & 6.7 & $0-27$ \\
\hline Current exacerbation (months) & 4.0 & $0-46$ & 7.6 & $0-98$ & 5.5 & $0-65$ \\
\hline Previous resection $(\mathrm{Y} / \mathrm{N})$ & & $28 / 30$ & & $27 / 34$ & & $34 / 24$ \\
\hline Time since resection (years) & 5.8 & $0-22$ & 5.3 & $0-23$ & 4.6 & $0-13$ \\
\hline
\end{tabular}




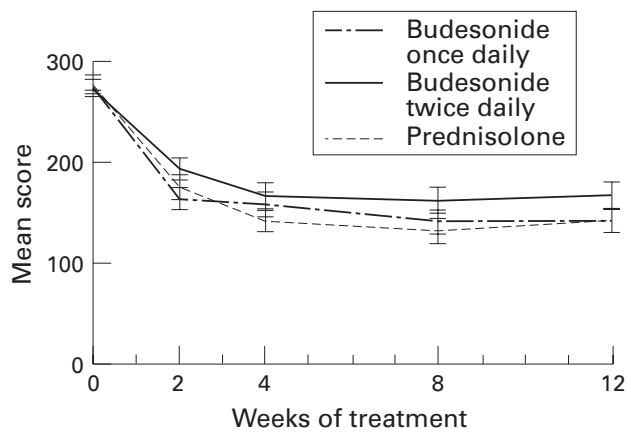

Figure 2: Mean (SE) CDAI score at randomisation and after two, four, eight, and 12 weeks of treatment with budesonide or prednisolone.

ment, were not significantly different $(p=0.80$, $\mathrm{p}=0.15)$.

\section{CDAI change}

The mean initial CDAI score was 277 for the budesonide once daily group, 274 for the budesonide twice daily group, and 279 for the prednisolone group. The most pronounced decrease in CDAI score in all three groups was observed during the first two treatment weeks.

As reflected by remission rates, the mean CDAI scores decreased more in the budesonide once daily group and prednisolone group than in the budesonide twice daily group. The difference between the groups in reduction of CDAI score was statistically significant after two weeks $(p=0.050)$ but not after eight weeks $(\mathrm{p}=0.093)($ fig 2$)$.

\section{SAFETY RESULTS}

Adverse events

Adverse events (any unfavourable eventssuch as clinical signs, symptoms, changes in laboratory data-temporarily associated with administration of the study drug) were registered in $78 \%$ of patients in the budesonide once daily group, $90 \%$ in the budesonide twice daily group, and $90 \%$ in the prednisolone group. Most adverse events were related to the gastrointestinal system, probably reflecting the underlying disease. A slightly higher frequency of dyspepsia was observed in the budesonide once daily group, while nausea and epigastric pain were more frequent in the budesonide twice daily group. The highest frequency of patients with Cushingoid features was observed in the prednisolone group. Four patients in the budesonide once daily group reported rashes compared with none in the other groups; the frequency of depression and insomnia, palpitations, and flushing was higher in the prednisolone group. The number of patients with urinary tract infections was higher in the budesonide twice daily group whereas increased frequency of micturition was reported only by prednisolone treated patients.

Eighteen adverse events in 17 patients, of which 10 discontinued study treatment, resulted in hospitalisation and were classified as serious. The majority of admissions were for disease deterioration or complications of Crohn's disease. A relationship between these serious adverse events and the study drug was judged, by the investigator, to be unlikely.
There was a significant difference between the three groups with respect to change in weight: after eight weeks, mean body weight increased by $1.0 \mathrm{~kg}$ in the budesonide once daily group and by $2.1 \mathrm{~kg}$ in the prednisolone group, but not at all in the budesonide twice daily group $(\mathrm{p}<0.0001)$.

\section{Haematology, clinical chemistry, and} inflammatory indicators

Most of the laboratory values found outside normal reference ranges were considered by the investigators to be related to the underlying Crohn's disease. There were no statistically significant differences between the three groups with respect to changes in the inflammatory indicators (ESR, serum CRP, serum orosomucoid).

Comparison of the mean changes in haematological and clinical chemistry variables from baseline showed a significant difference $(p=0.029)$ at 12 weeks between the groups with respect to leucocyte count. After 12 weeks the mean leucocyte count in the prednisolone group significantly increased by $0.9 \times 10^{9} / 1$; it decreased by $0.5 \times 10^{9} / 1$ in the budesonide once daily group, and very slightly increased by $0.1 \times 10^{9} / 1$ in the budesonide twice daily group. No other haematological and clinical chemistry variables differed significantly between the groups.

\section{Basal plasma cortisol}

The mean plasma cortisol values at randomisation were similar in the groups-that is, $382 \mathrm{nmol} / 1$ in the budesonide once daily group, $374 \mathrm{nmol} / \mathrm{l}$ in the budesonide twice daily group, and $375 \mathrm{nmol} / \mathrm{l}$ in the prednisolone group. There was a decrease in all three groups during the treatment period (fig 3). After eight weeks of treatment the mean plasma cortisol value had decreased by $258 \mathrm{nmol} / 1$ in the prednisolone group, by $194 \mathrm{nmol} / 1$ in the budesonide once daily group, and by $132 \mathrm{nmol} / \mathrm{l}$ in the budesonide twice daily group. The difference between the groups was statistically significant $(p=0.0035)$. There was no significant difference between the two budesonide groups $(p=0.096)$. Mean plasma cortisol values after two, eight, and 12 weeks were always lower in the prednisolone group.

The proportion of patients with values below the lower plasma cortisol normal reference limit - $150 \mathrm{nmol} / \mathrm{l}$ — was significantly higher in

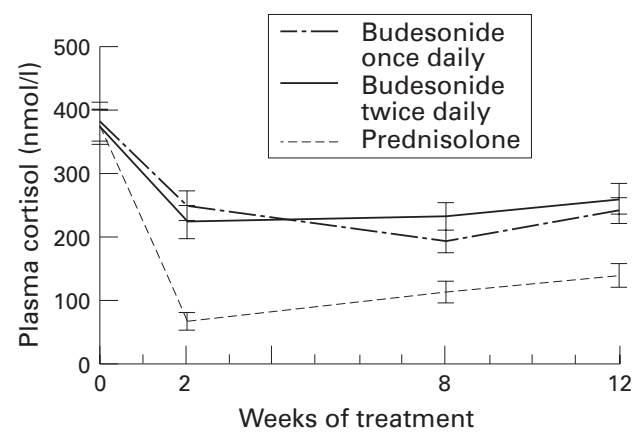

Figure 3: Mean (SE) morning plasma cortisol at randomisation and after two, four, eight, and 12 weeks of treatment with budesonide or prednisolone. 
TABLE 2 Adrenal function (short ACTH test) before and after treatment

\begin{tabular}{llll}
\hline Treatment & $\begin{array}{l}\text { At randomisation } \\
(\%)\end{array}$ & $\begin{array}{l}\text { After 8 weeks } \\
(\%)\end{array}$ & $\begin{array}{l}\text { Comparisons } \\
\text { after 8 weeks }\end{array}$ \\
\hline Budesonide once daily & 86 & 42 & $\begin{array}{l}\mathrm{p}=0.55^{\star} \\
\mathrm{p}=0.013 \dagger \\
\text { Budesonide twice daily }\end{array}$ \\
\begin{tabular}{l} 
Prednisolone \\
\hline
\end{tabular} & 90 & 50 & $\mathrm{p}=0.0015 \ddagger$ \\
\hline
\end{tabular}

^Versus budesonide twice daily; tversus prednisolone; łversus prednisolone.

the prednisolone group compared with both budesonide groups. After eight weeks, $76 \%$ of prednisolone treated patients had plasma cortisol values below $150 \mathrm{nmol} / 1$ compared with $41 \%$ in the budesonide once daily group $(\mathrm{p}=0.0004)$ and $36 \%$ in the budesonide twice daily group $(\mathrm{p}<0.0001)$.

Analysis of adrenal function (short ACTH test) revealed statistically significant differences between the groups at eight weeks $(p=0.0023)$ (table 2). After eight weeks, the proportion of patients with normal adrenal function was reduced in all three groups. The maximum reduction was found in the prednisolone group, the difference versus both budesonide once daily $(p=0.013)$ and budesonide twice daily $(p=0.0015)$ being significant. There was no significant difference between the two budesonide groups in this respect.

Glucocorticoid associated side effects

The proportion of patients with glucocorticoid associated side effects was not significantly different between the three groups: $50 \%$ in the budesonide once daily group, $44 \%$ in the budesonide twice daily group, and $59 \%$ in the prednisolone group. However, the number of patients with moon faces found in the prednisolone group was approximately three times higher than in the budesonide groups $(p=0.0005)$. The difference between the groups with respect to other GCS associated side effects was also significant $(p=0.0098)$. Table 3 presents a summary of side effects.

\section{Discussion}

Although corticosteroid therapy represents the keystone approach for treating patients with active Crohn's disease, its therapeutic value is counterbalanced by a number of side effects related to systemic activity and to suppression of endogenous adrenal function with associated long term problems and, rarely, idiosyn-

TABLE 3 Glucocorticoid associated side effects

\begin{tabular}{|c|c|c|c|c|c|c|}
\hline \multirow[b]{2}{*}{ Sign } & \multicolumn{2}{|c|}{ Budesonide once daily } & \multicolumn{2}{|c|}{ Budesonide twice daily } & \multicolumn{2}{|c|}{ Prednisolone } \\
\hline & $\begin{array}{l}\text { Before } \\
\text { study }\end{array}$ & $\begin{array}{l}\text { During } \\
\text { study }\end{array}$ & $\begin{array}{l}\text { Before } \\
\text { study }\end{array}$ & $\begin{array}{l}\text { During } \\
\text { study }\end{array}$ & $\begin{array}{l}\text { Before } \\
\text { study }\end{array}$ & $\begin{array}{l}\text { During } \\
\text { study }\end{array}$ \\
\hline Moon face & 1 & 8 & 2 & 7 & 2 & 22 \\
\hline Acne & 1 & 12 & 6 & 11 & - & 11 \\
\hline Swollen ankles & - & 5 & - & 2 & - & 3 \\
\hline Bruises easily & 5 & 7 & 4 & 10 & 2 & 7 \\
\hline Hirsutism & 1 & 3 & 1 & 3 & 2 & 3 \\
\hline Buffalo hump & - & - & - & - & - & 2 \\
\hline Skin striae & - & - & 1 & - & - & - \\
\hline Others ${ }^{\star}$ & - & 4 & - & 9 & 1 & 16 \\
\hline
\end{tabular}

Some patients experienced more than one glucocorticocoid associated side effect.

${ }^{\star}$ Symptoms considered by the investigator to be signs of possible adverse effects were: weight increase, sweating, dyspepsia, nausea, stiff joints, headache, depression, insomnia, weakness, irritated facial skin, mood swings, limb discomfort, hot flushes, sleep disorder, impaired healing, localised papules, mentally stimulated, cramps in calves, agitation, irritability, emotional lability, generalised oedema, palpitations, localised erythema, facial oedema, and epigastric pain. cratic or allergic reactions. The possibility of using a second generation of corticosteroids with comparable efficacy but with fewer side effects offers the prospect of a safer therapy.

Budesonide was shown to be active when given in rectal enemas to patients with ulcerative colitis. An early study showed that it was better than placebo, and other trials have demonstrated that it was comparable to prednisolone in its efficacy but with significantly less action on the pituitary adrenal axis. ${ }^{4-9}$ The CIR formulation was devised to treat patients with active Crohn's disease localised to the ileum or the ascending $\operatorname{colon}^{10}$ and the value of this formulation has been tested in two trials. ${ }^{12}{ }^{13}$ A placebo controlled dose finding study $^{12}$ suggested that $9 \mathrm{mg}$ daily ( $4.5 \mathrm{mg}$ twice daily) is the minimal effective dosage of budesonide. In the second study, ${ }^{13}$ budesonide $9 \mathrm{mg}$ once daily was as effective as prednisolone $40 \mathrm{mg}$ once daily in inducing remission; at eight weeks, $52 \%$ of patients in the budesonide group were in remission compared with $65 \%$ in the prednisolone group $(p=0.12)$. The purpose of the present study was, therefore, to compare the two different dose regimens of budesonide CIR therapy - a single morning dose versus a twice daily dosage - and these two approaches were again compared with the standard prednisolone regimen of $40 \mathrm{mg}$ daily, with special reference to efficacy and effects on adrenal axis function. After two weeks of treatment, no significant differences in clinical response were observed between the prednisolone and budesonide once daily groups but fewer remissions were observed in the budesonide twice daily group. After eight weeks, equal remission rates were obtained in the prednisolone and budesonide once daily groups and a somewhat lower remission rate with budesonide twice daily.

The CDAI scores for patients on prednisolone or budesonide once daily decreased in a similar fashion, with a less rapid decline in the budesonide twice daily group. As one of the first aims in treating patients with inflammatory bowel disease is the prompt disappearance of symptoms, this goal was most clearly achieved with budesonide once daily and prednisolone within the first two weeks. These results confirm that budesonide $9 \mathrm{mg}$ daily, given as a single morning dose, is as effective as $40 \mathrm{mg}$ prednisolone, as indicated in the previous study. ${ }^{13}$ As we found that budesonide was associated with much less impairment of adrenal axis function, this treatment may well represent the first choice for the management of patients with active Crohn's disease.

Patients with CDAI > 300 showed generally a weaker response to treatment compared with those with CDAI <300. In the former group, a higher remission rate was obtained with prednisolone compared with the two budesonide treatments $(54 \%, 31 \%$, and $17 \%$ respectively). This trend is not statistically significant $(p=0.07)$ but it might indicate that corticosteroids with systemic effects have a specific role in the treatment of the most severe cases of Crohn's disease. However, even in this subgroup, budesonide would be an important 
alternative for patients in whom systemically active steroids should be avoided, such as diabetics.

In the previous comparative study of budesonide $9 \mathrm{mg}$ daily versus prednisolone $40 \mathrm{mg}$ daily, CDAI remission rates at two, four, and eight weeks always favoured prednisolone, and were significant at four weeks $(67 \% v 40 \%$, $\mathrm{p}<0.001) .{ }^{13}$ However, in the present study, the highest remission rate occurred with budesonide once daily after two weeks; at eight weeks, budesonide once daily did as well as prednisolone. It is difficult to explain the difference between our findings and those of the previous study. There was no substantial difference in severity of the study groups as judged by CDAI scores, and in both studies a single morning dose of budesonide was used. With regard to the different rates of remission observed in the budesonide once daily and the budesonide twice daily groups, it seems that a pulsed dosage regimen produces a more powerful effect. ${ }^{18} 19$ As a once daily approach is the most practical and acceptable way to administer a drug to patients and may achieve better compliance, the single morning administration can be recommended. Evidence of adrenal axis suppression was significantly greater in the prednisolone treated patients than in budesonide treated patients. Prednisolone treated patients also showed significant increases in peripheral leucocyte counts and other effects associated with the systemic action of corticosteroids. The conclusions of our multicentre trial are:

- Budesonide CIR, administered as a single daily dosage of $9 \mathrm{mg}$ daily or $4.5 \mathrm{mg}$ twice daily, is comparable to prednisolone for the induction of remission in patients with active Crohn's disease.

- The single morning administration of budesonide CIR is as promptly effective as prednisolone and represents a simpler and safer therapeutic approach, with a reduction in side effects. ${ }^{20}$

- Budesonide CIR offers a useful advance in the treatment of active Crohn's disease while we await a new breakthrough in the therapy of this challenging disease. ${ }^{21}$

\section{Appendix}

Members of the Global Budesonide Study Group are: $\mathrm{H}$ Malchow, Medizinische Klinik II, Leverkusen, Germany; C Prantera, Department of Gastroenterology, Ospedale "Nuovo Regina Margherita", Rome, Italy; V Mani, Leigh Infirmary, Leigh, UK; C O'Morain, Meath Hospital, Dublin, Ireland; W Selby, Royal Prince Alfred Medical Centre, Newtown, Australia; F Pallone, II Clinica MedicaPoliclinico, Rome, Italy; M Mazzetti di Pietralata, S Eugenio Hospital, Rome, Italy; R Sjödahl, University Hospital, Linköping, Sweden; T Florin, Mater Adult Hospital, Australia; P Smith, Llandough Hospital, South Glamorgan, UK; P Bianchi, Instituto di Clinica Medica I, Milan, Italy; R Löfberg, Huddinge
Hospital, Sweden; P Rutgeerts, University of Leuven, Belgium; R Smallwood, Repatriation General Hospital, Heidelberg, Australia; C B $\mathrm{H}$ W Lamers, University Hospital, Leiden, The Netherlands; C Tasman-Jones, Auckland University School of Medicine, Auckland, New Zealand; J O Hunter, Addenbrooke's Hospital, Cambridge, UK; H Hodgson, Hammersmith Hospital, London, UK; $\AA$ Danielsson, University Hospital, Umeå, Sweden; F I Lee, Victoria Hospital, Blackpool, UK; G Piacitelli, Hospital S Giovanni, Rome, Italy; A Ellis, Broadgreen Hospital, Liverpool, UK; D G Weir, St James' Hospital, Dublin, Ireland.

This study was supported by a grant from Astra Draco AB, Sweden.

1 Podolsky DK. Inflammatory bowel disease. $N$ Engl f Med 1991; 325: 1008-16.

2 Meyers S, Sachar DB. Medical management of Crohn's disease. Hepatogastroenterology 1990; 37: 42-55.

3 Summers RW, Switz DM, Sessions JT Jr, Becktel JM, Best WR, Kern F, et al. National Cooperative Crohn's Disease Study: results of drug treatment. Gastroenterology 1979; 77: 847-69.

4 Brattsand R. Overview of newer glucocorticoid preparations for inflammatory bowel disease. Can f Gastroenterol 1990; 4: 407-14.

5 Brogden RN, McTavish D, Barnes P J, Gross NJ, Juniper E, Laursen LC, et al. Budesonide: an updated review of its pharmacological properties, and therapeutic efficacy in asthma and rhinitis. Drugs 1992; 44: 375-407.

6 The Danish Budesonide Study Group. Budesonide enema in distal ulcerative colitis: a randomized dose-response trial with prednisolone enema as positive control. Scand $\mathcal{f}$ Gaswith prednisolone enema as posterol 1991; 26: 1225-30.

7 Löfberg R, Østergaard-Thomsen O, Langholz E, Schioeler $\mathrm{R}$, Danielsson AA, Suhr O, et al. Budesonide versus prednisolone retention enema in active distal ulcerative colitis. Aliment Pharmacol Ther 1994; 8: 623-9.

8 Bianchi Porro G, Prantera C, Campieri M, Petrillo M, Campanini MC, Gionchetti P, et al. Comparative trial of methylprednisolone and budesonide enema in active distal ulcerative colitis. Eur f Gastroenterol Hepatol 1994; 6: $125-$ 30.

9 Tarpila S, Turunen U, Seppälä K, Aukee S, Pikkarainen P, Elomaa I, et al. Budesonide enema is as effective as hydro8: $591-5$.

10 Edsbäcker S, Wollmer P, Nilsson Å, Nilsson M. Pharmacokinetics and gastrointestinal transit of budesonide
controlled ileal release (CIR) capsules. Gastroenterology 1993; 104: A695.

11 Löfberg R, Danielsson Å, Salde L. Oral budesonide in active Crohn's disease. Aliment Pharmacol Ther 1993; 7: 611-6.

12 Greenberg GR, Feagan BG, Martin F, Sutherland LR, Thomson AB, Williams CN, et al. Oral budesonide for active Crohn's disease. N Engl f Med 1994; 331: 836-41.

13 Rutgeerts P, Löfberg R, Malchow H, Lamers C, Olaison G, Jewell D, et al. A comparison of budesonide with prednisolone for active Crohn's disease. $N$ Engl f Med 1994; 331: 842-5

14 Best WR, Becktel JM, Singleton JW, Kern F. Development of a Crohn's disease activity index. National Cooperative Crohn's Disease Study. Gastroenterology 1976; 70: 439-44.

15 Ögren L, Jönsson P. Determination of cortisol in human plasma by solid phase extraction and coupled column
liquid chromatography. Astra Draco Report 850-RD-0323, 1994.

16 Malchow H, Ewe K, Brandes JW, Goebell H, Ehms H, Sommer H, et al. European Cooperative Crohn's Disease Study (ECCDS): resuls
ogy 1984; 86: 249-66.

17 Hanauer SB, Stathopoulos G. Risk-benefit assessment of drug used in the treatment of inflammatory bowel disease. Drug Saf 1991; 6: 192-219.

18 Reinberg A, Smolensky MH, D'Alonzo GE, Mc Govern JP. Chronobiology and asthma III. Timing corticotherapy to biological rhythms to optimize treatment goals. F Asthma 1988; 25: 219-48.

19 Faucy AS, Dale DC, Balow JE. Glucocorticoid therapy: mechanisms of action and clinical considerations. Ann Intern Med 1976; 84: 304-15.

20 Edsbäcker S, Larsson P, Nilsson M, Wirén JE. Budesonide controlled ileal release (CIR) capsules affect plasma cortisol less than prednisolone. Gastroenterology 1995; 108: A814.

21 Sachar DB. Budesonide for inflammatory bowel disease: is it a magic bullet? N Engl f Med 1994; 331: 873-4. 